\section{KVen treiben Spielchen mit neuen Geriatrieleistungen}

\begin{abstract}
— Seit dem 1. Juli 2016 gibt es neue Geriatrieleistungen im EBM. Zusätzlich zu den alten Nrn. 03360 und 03362 gibt es nun die Nr. 30984 für das spezielle geriatrische Assessment, das von Spezialisten erbracht wird. Für Hausärzte interessant sind die vorherige Abklärung nach Nr. 30980 (194 Punkte) und die Nr. 30988 (65 Punkte), die der Nr. 03362 zugeschlagen werden kann, wenn ein Patient vom speziellen Assessment zurückkommt (siehe MMW-Sonderheft 1/2016 S. 16). Diese neuen Leistungen sollen ex-
\end{abstract}

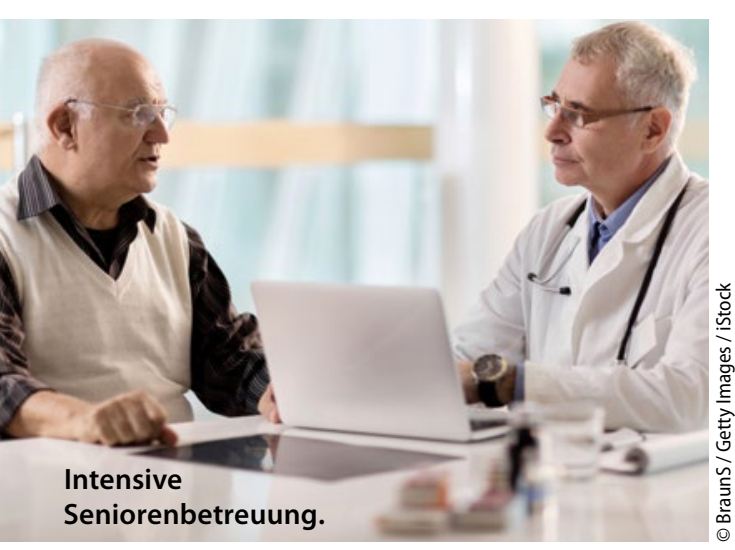

trabudgetär in Euro vergütet werden, was bei den alten Leistungen nicht der Fall ist. In vielen KVen wird aber rechtswidrig beides budgetiert.

\section{MMW-KOMMENTAR}

Der Bewertungsausschuss von Kassen und KBV hat am 31. Mai 2013 beschlossen, dass u. a. die Versorgung geriatrischer Erkrankungen und Behinderungen mit neuen EBM-Nrn. gefördert werden soll. Das mit den Kassen vereinbarte zusätzliche Honorarvolumen muss dabei aber für die gezielte Verbesserung dieser Versorgungsbereiche verwendet werden. Eine Honorarverteilung ist also nur rechtskonform, wenn für die Leistungen ein eigener "Topf" gebildet wird. Eine Honorierung innerhalb des Regelleistungsvolumens (RLV) wird dieser eindeutigen Vorgabe nicht gerecht.

Auch die Bildung eines qualifikationsgebundenen Zusatzvolumens (QZV) für geriatrische und palliativmedizinische Leistungen ist unzulässig, da QZV auf der Grundlage eines Fallwerts bemessen werden. Dass heißt, dass bei der Ermittlung des praxisindividuellen Budgets in Euro alle Fälle der Praxis zugrun-

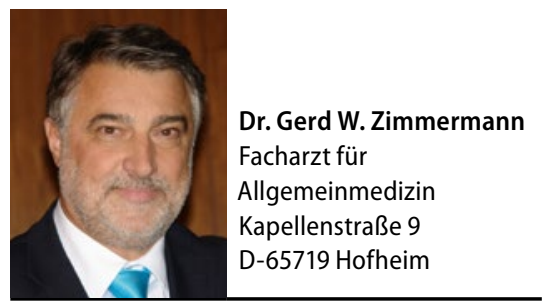

de gelegt werden - und nicht nur die genau definierten geriatrischen und palliativmedizinischen. Das führt dazu, dass Praxen mit eher niedrigen Fallzahlen und einem hohen Anteil an geriatrischen Patienten die erbrachten Leistungen nur zu einer sehr geringen Quote vergütet bekommen. Dagegen erhalten Praxen mit hohen Fallzahlen und ggf. wenigen geriatrischen Patienten ein sehr hohes QZV. Dieses wiederum schöpfen sie nicht aus, der Rest wird auf ihr RLV übertragen und fördert somit die Versorgung nicht-geriatrischer Fälle. Das war aber sicher nicht die Intention hinter dem Beschluss.

Der Auftrag des Bewertungsausschusses kann nur umgesetzt werden, wenn das QZV nur anhand der im EBM definierten Fälle der Geriatrie oder Palliativmedizin gebildet wird. Eine andere Möglichkeit wäre noch, einfach beide Bereiche im Honorarverteilungsmaßstab als "freie Leistungen" darzustellen und mit dem vereinbarten Honorarvolumen auszustatten.

Geht eine KV anders vor, sollte dies Anlass zu einem Widerspruch gegen die Honorarmitteilung sein. Lehnt die KV diesen ab, kann das Sozialgericht angerufen werden.

\title{
Hausärzte arbeiten mehr für weniger Geld
}

_ Der Honorarreport der KBV für das 4. Quartal 2014 zeigt, dass Hausärzte durchschnittlich 56 Stunden pro Woche in der Praxis tätig sind - Fachärzte aber nur 53. Der Honorarzuwachs der Vertragsärzte fiel mager aus: Der Umsatz je Arzt stieg im Schnitt um 348 Euro (+0,7\%) auf 52.234 Euro, der Umsatz je Behandlungsfall um 48 Cent auf 62,39 Euro (+0,8\%).

\section{MMW-KOMMENTAR}

Im hausärztlichen Bereich stieg der Durchschnittswert im Vergleich zum Vorjahresquartal je Arzt um 2,3\% auf 53.182 Euro und je Behandlungsfall um 1,4\% auf 62,11 Euro. Hier gibt es aber starke regionale Unterschiede: Die Ärzte in Hamburg konnten sich über einen Zuwachs pro Arzt um 6,4\% freuen, in Thüringen gab es ein Minus von 3,4\%. Auch in Berlin, Rheinland-Pfalz und Mecklenburg-
Vorpommern gab es weniger Geld. Betrachtet man nur die Allgemeinmediziner und Hausarzt-Internisten - rechnet man also die Kinder- und Jugendärzte heraus - liegt das Plus sogar nur bei 2,0\% je Arzt bzw. 1,3\% je Behandlungsfall.

Im fachärztlichen Bereich gab es pro Fall nur 0,3\% mehr als 2013 - der Fallwert von durchschnittlich 66,61 Euro übertrifft den der Hausärzte aber weiter um satte $8,4 \%$. 